\title{
AN ANALYTICAL STUDY ON BUCKLING BEHAVIOR OF CNT/POLYMER COMPOSITE PLATES USING THE FIRST ORDER SHEAR DEFORMATION THEORY
}

\author{
Pramod Kumar Peyyala, V.V. Subba Rao \\ Jawaharlal Nehru Technological University Kakinada, India \\ e-mail: pramod.330@gmail.com; rao703@yahoo.com
}

\begin{abstract}
Most plates used in engineering structures such as aircraft wings, ship ducts and buildings, although quite capable of resisting tensile loads, are poor in withstanding compression. In order to avoid premature failure under compression, it is important to know buckling behavior of the plate. This article primarily deals with the analytical study of buckling behavior of a carbon nanotube reinforcing polymer composite plates based on the first order shear deformation theory by employing Mori-Tanaka micromechanics approach to obtain elastic properties. In this investigation, an attempt is made for evaluating the effect of plate thickness, CNT volume fraction, stacking sequence and CNT radii on the buckling of plates.
\end{abstract}

Keywords: buckling, carbon nanotube, composite plates, micromechanics, FSDT

\section{Introduction}

Since the recognized discovery of Carbon Nanotubes (CNTs), Iijima (1991), they have attracted an intense interest among researchers in various disciplines due to their stupendous mechanical, electrical and thermal properties (Saito et al., 1999; Ounaies et al., 2003; Weisenberger et al., 2003). The remarkable electrical properties have made CNTs excellent candidates to act as reinforcement in a wide range of applications such as nanosensors and atomic transportation (Arash and Wang, 2013; Wang, 2008). In addition, the outstanding physical and mechanical properties of CNTs, for example an ultra-high Young's modulus of the order of $1 \mathrm{TPa}$ and tensile strength of $200 \mathrm{GPa}$ (Lau and Hui, 2002; Demczyk et al., 2002), stimulated the interest in using CNTs as filler materials in polymer composites to make ultra light structural materials with enhanced mechanical properties.

In the past decade, the wide-ranging experimental and theoretical studies conducted on carbon nanotubes have focused on mechanical characterization and modeling aspects of reinforced polymer composites to assist the development of nanocomposites (Xiao et al., 2005; Zhang Z. et al., 2010; Zhang Y. et al., 2013; Arash et al., 2014; Silani et al., 2014; Gates et al., 2005; Tserpes and Papnikos, 2005). Even though these studies are fairly useful in estimating properties of the nanomaterials, their use in actual structural applications is the ultimate idea behind the development of this highly sophisticated class of materials. However, the literature shows that much of work in this direction is not accomplished. As such, there is a need to examine the macro behavior of the material in actual structural elements such as beams and plates. The behavior of beams under a static loading was studied by Wuite and Adali (2005) for different CNT volume of fractions and nanotube diameters. Based on different assumptions for displacement fields, different theories for plate analysis have been devised. These theories can be divided into three major categories, individual layer theories, equivalent single-layer (ESL) theories, and 
three-dimensional elasticity solution procedures. These categories are further divided into sub-theories by the introduction of different assumptions. For example, the second category includes the classical laminate plate theory (CLPT), the first-order and higher-order shear deformation theories (FSDT and HSDT). Analytical observations on static and dynamic behavior of plates by using CLPT were made by Madhu et al. (2012).

For the implementation of Carbon nanotube reinforced polymer (CNRP) composites in structural applications, accurate property-microstructure relations are required in the form of micromechanics models. In the present investigation, micromechanics properties of CNRP are computed using Mori-Tanaka method as given in (Popov et al., 2000; Shi et al., 2004; Wuite and Adali, 2005; Madhu et al., 2012). The effects of the characteristics developed in these models are investigated on the buckling behavior of CNRP composite plates using FSDT with a view towards assessing the effectiveness of these materials in the design of structural nanocomposites.

\section{Micro-mechanics model}

The micro-mechanical model involves an elastic and isotropic polymer reinforced with straight, aligned and infinitely longsingle walled carbon nanotubes (SWCNT). The plate under the study is composed of polystyrene as the matrix with the Young modulus and Poisson's ratio of $E_{m}=1.9 \mathrm{GPa}$ and $\nu_{m}=0.3$, respectively. Each SWCNT is considered to be solid and transversely isotropic, and their Hill's elastic constants are taken from Popov et al. (2000). The SWCNT radius is assumed to be $10 \AA$ for all the cases, otherwise mentioned, for which the representative values of the elastic constants are: $n_{r}=450 \mathrm{GPa}, k_{r}=30 \mathrm{GPa}, m_{r}=p_{r}=1 \mathrm{GPa}$ and $l_{r}=10 \mathrm{GPa}$. The bonding at the nanotube-polymer interface is taken to be perfect. The resulting composite is also considered as transversely isotropic and its constitutive stress strain relations $\sigma=C \varepsilon$ can be expressed as follows

$$
\left\{\begin{array}{l}
\sigma_{11} \\
\sigma_{22} \\
\sigma_{33} \\
\sigma_{23} \\
\sigma_{13} \\
\sigma_{12}
\end{array}\right\}=\left[\begin{array}{cccccc}
(n & l & l & 0 & 0 & 0 \\
l & k+m & k-m & 0 & 0 & 0 \\
l & k-m & k+m & 0 & 0 & 0 \\
0 & 0 & 0 & 2 m & 0 & 0 \\
0 & 0 & 0 & 0 & 2 p & 0 \\
0 & 0 & 0 & 0 & 0 & 2 p
\end{array}\right]\left\{\begin{array}{l}
\varepsilon_{11} \\
\varepsilon_{22} \\
\varepsilon_{33} \\
\varepsilon_{23} \\
\varepsilon_{13} \\
\varepsilon_{12}
\end{array}\right\}
$$

where $k$ is the plane-strain bulk modulus normal to the fiber direction, $n$ is the uni-axial tension modulus in the fiber direction (1), $l$ is the associated cross modulus, $m$ and $p$ are the shear moduli in planes normal and parallel to the fiber direction, respectively, and they are Hill's elastic constants. A composite with a reinforcing phase volume fraction $c_{r}$, matrix Young's modulus $E_{m}$ and matrix Poisson's ratio $\nu_{m}$ is considered. Using the Mori-Tanaka method, Hill's elastic moduli are found to be (Popov et al., 2000; Shi et al., 2004; Wuite and Adali, 2005; Madhu et al., 2012)

$$
\begin{aligned}
k= & \frac{E_{m}\left\{E_{m} c_{m}+2 k_{r}\left(1+\nu_{m}\right)\left[1+c_{r}\left(1-2 \nu_{m}\right)\right]\right\}}{2\left(1+\nu_{m}\right)\left[E_{m}\left(1+c_{r}-2 \nu_{m}\right)+2 c_{m} k_{r}\left(1-\nu_{m}-2 \nu_{m}^{2}\right)\right]} \\
l= & \frac{E_{m}\left\{c_{m} \nu_{m}\left[E_{m}+2 k_{r}\left(1+\nu_{m}\right)\right]+2 c_{r} l_{r}\left(1-\nu_{m}^{2}\right)\right\}}{\left(1+\nu_{m}\right)\left[2 c_{m} k_{r}\left(1-\nu_{m}-2 \nu_{m}^{2}\right)+E_{m}\left(1+c_{r}-2 \nu_{m}\right)\right]} \\
n= & \frac{E_{m}^{2} c_{m}\left(1+c_{r}-c_{m} \nu_{m}\right)+2 c_{m} c_{r}\left(k_{r} n_{r}-l_{r}^{2}\right)\left(1+\nu_{m}\right)^{2}\left(1-2 \nu_{m}\right)}{\left(1+\nu_{m}\right)\left[2 c_{m} k_{r}\left(1-\nu_{m}-2 \nu_{m}^{2}\right)+E_{m}\left(1+c_{r}-2 \nu_{m}\right)\right]} \\
& +\frac{E_{m}\left[2 c_{m}^{2} k_{r}\left(1-\nu_{m}\right)+c_{r} n_{r}\left(1-2 \nu_{m}+c_{r}\right)+4 c_{m} c_{r} l_{r} \nu_{m}\right]}{2 c_{m} k_{r}\left(1-\nu_{m}-2 \nu_{m}^{2}\right)+E_{m}\left(1+c_{r}-2 \nu_{m}\right)}
\end{aligned}
$$




$$
\begin{aligned}
& p=\frac{E_{m}\left[E_{m} c_{m}+2\left(1+c_{r}\right) p_{r}\left(1+\nu_{m}\right)\right]}{2\left(1+\nu_{m}\right)\left[E_{m}\left(1+c_{r}\right)+2 c_{m} p_{r}\left(1+\nu_{m}\right)\right]} \\
& m=\frac{E_{m}\left[E_{m} c_{m}+2 m_{r}\left(1+\nu_{m}\right)\left(3+c_{r}-4 \nu_{m}\right)\right]}{2\left(1+\nu_{m}\right)\left\{E_{m}\left[c_{m}+4 c_{r}\left(1-\nu_{m}\right)\right]+2 c_{r} m_{r}\left(3-\nu_{m}-4 \nu_{m}^{2}\right)\right\}}
\end{aligned}
$$

where $k_{r}, l_{r}, m_{r}, n_{r}$ and $p_{r}$ are Hill's elastic moduli for the reinforcing phase. The expressions for the moduli of the CNTRC as functions of the stiffness constants are determined for a unidirectional composite as follows

$$
E_{1}=n-\frac{l^{2}}{k} \quad E_{2}=\frac{4 m\left(k n-l^{2}\right)}{k n-l^{2}+m n} \quad G_{12}=2 p \quad \nu_{12}=\frac{l}{2 k}
$$

\section{Basic equations}

The buckling behavior of a symmetric laminated carbon nanotube reinforced polymer square plate is to be studied under the first order shear deformation theory (FSDT), and the basic equations are summarized in this section.

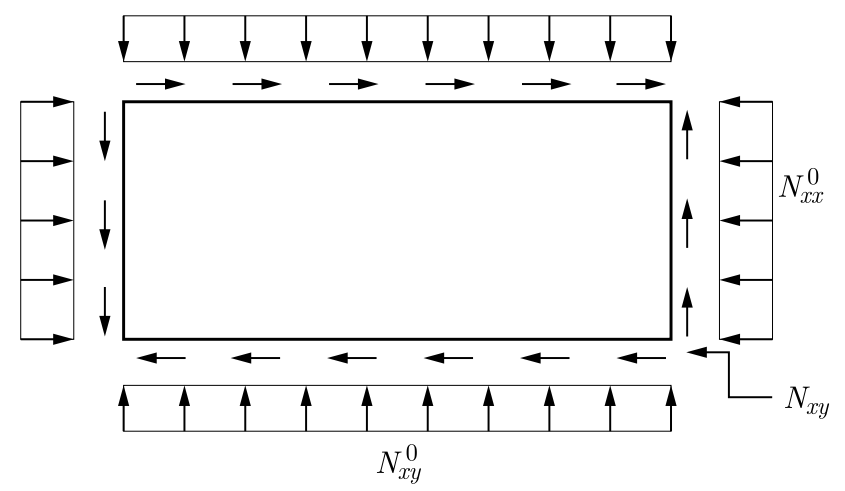

Fig. 1. Buckling of a plate under in-plane compressive edge forces

For laminated composite plates subjected to only in-plane loads, the constitutive relations for the buckling under FSDT are taken from Reddy (2004). A parametric study is carried out to find the minimum buckling load for laminated composite plates which occurs at $m=n=1$. In the buckling analysis, it is assumed that the loads are in-plane forces only.

For simplicity

$$
\widehat{N}_{x x}=-N_{0} \quad \widehat{N}_{y y}=-k N_{0} \quad k=\frac{\widehat{N}_{y y}}{\widehat{N}_{x x}}
$$

Therefore:

— for uniaxial compression $k=0$

$$
\widehat{N}_{x x}=-N_{0} \quad \widehat{N}_{y y}=0
$$

— for biaxial compression $k=1$

$$
\widehat{N}_{x x}=-N_{0} \quad \widehat{N}_{y y}=-N_{0}
$$

The critical buckling load is nondimensionalised by using

$$
\bar{N}=N_{c r} \frac{a^{2}}{E_{2} h^{2}}
$$

here $\bar{N}$ is the nondimensionalised critical buckling load, $N_{c r}$ is the critical buckling load, $a$ is length of the plate, $E_{2}$ is Young's modulus in the transverse direction and $h$ is thickness of the laminated composite plate. 


\section{Buckling analysis}

A proficient MATLAB code is developed for the buckling analysis using FSDT of carbon nanotube reinforced polymer (CNRP) composite plates. A twofold validation is done to the code, one for validating the elastic properties of CNRP and the other for validating the critical buckling load of composite plates under FSDT. First, the elastic properties of CNRP obtained from Mori-Tanaka micromechanics model are validated with the published results (Wuite and Adali, 2005) and presented in Fig. 2.

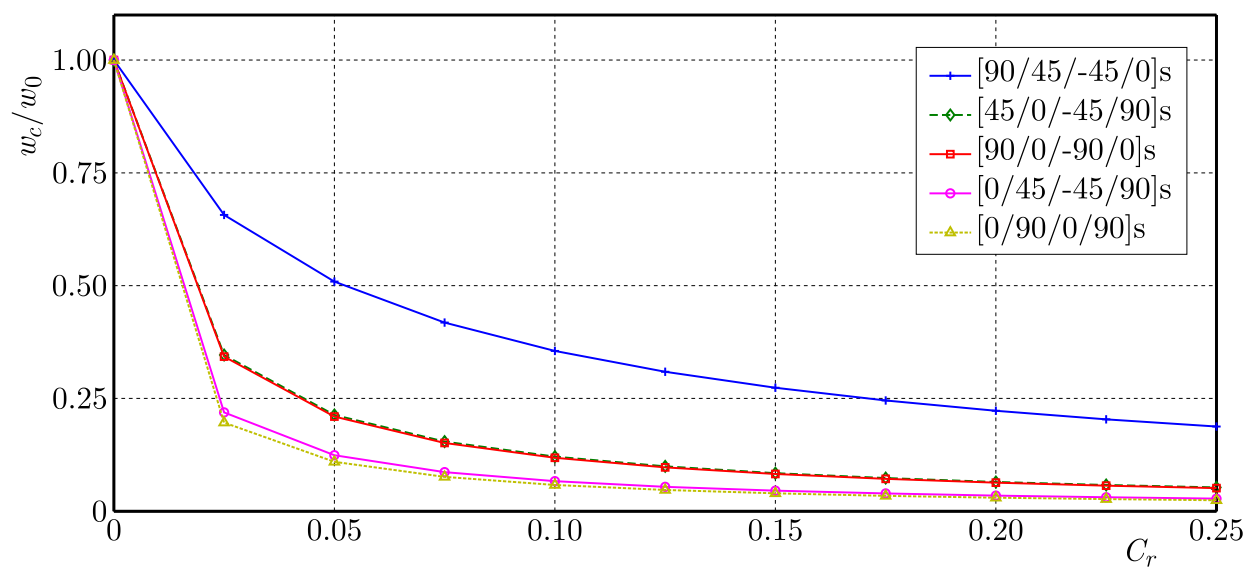

Fig. 2. Curves of the maximum deflection plotted against the fiber volume fraction for various stacking sequences for a simply supported beam subjected to $\mathrm{CPL}$

Further to validate the buckling with the published results, the effect of shear deformation on the nondimensionalised critical buckling loads $\bar{N}$ of symmetric laminated plates with elastic properties $E_{1}=25 E_{2}, G_{12}=G_{13}=0.5 E_{2}, G_{23}=0.2 E_{2}, \nu_{12}=0.25, K=5 / 6$ is calculated for uniaxial as well as biaxial compression and presented in Table 1 along with the corresponding published results (Reddy, 2004).

Table 1. Comparison of the nondimensionalised critical buckling load with the published results

\begin{tabular}{|c|c|c|c|c|}
\hline \multirow{2}{*}{$a / h$} & {$[0 / 90 / 0]$} & \multicolumn{2}{|c|}{$[0 / 90 / 0 / 90 / 0]$} \\
\cline { 2 - 5 } & $\bar{N}$ & Reddy $(2004)$ & $\bar{N}$ & Reddy $(2004)$ \\
\hline \hline \multicolumn{5}{|c|}{ Uniaxial compression } \\
\hline 10 & 15.248 & 15.289 & 16.254 & 16.309 \\
\hline 20 & 20.602 & 20.628 & 21.094 & 21.125 \\
\hline 25 & 21.552 & 21.568 & 21.893 & 21.917 \\
\hline 50 & 22.971 & 22.978 & 23.071 & 23.078 \\
\hline 100 & 23.361 & 23.363 & 23.384 & 23.389 \\
\hline \multicolumn{5}{|c|}{ Biaxial compression } \\
\hline 10 & 7.569 & 7.644 & 8.070 & 8.154 \\
\hline 20 & 10.293 & 10.314 & 10.522 & 10.562 \\
\hline 25 & 10.771 & 10.784 & 10.930 & 10.958 \\
\hline 50 & 11.486 & 11.489 & 11.531 & 11.539 \\
\hline 100 & 11.681 & 11.682 & 11.692 & 11.695 \\
\hline
\end{tabular}

The results obtained are found to be in excellent agreement with the published results. Having validated the composite plate results, the MATLAB code is extended to the CNRP 
composite plate to make an attempt to analyze the buckling behavior of these plates under FSDT.

An eight-layer simply supported symmetric square CNRP composite plate is subjected to both uniaxial and biaxial compression to study the buckling behavior. For the CNRP composite plate, the critical buckling load occurrs at $m=n=1$, and the variation in critical buckling load with respect to side-to-thickness ratio $a / h$ of the plate and CNT volume fraction (fiber volume fraction) for various stacking sequences is shown in Figs. 3 and 4.

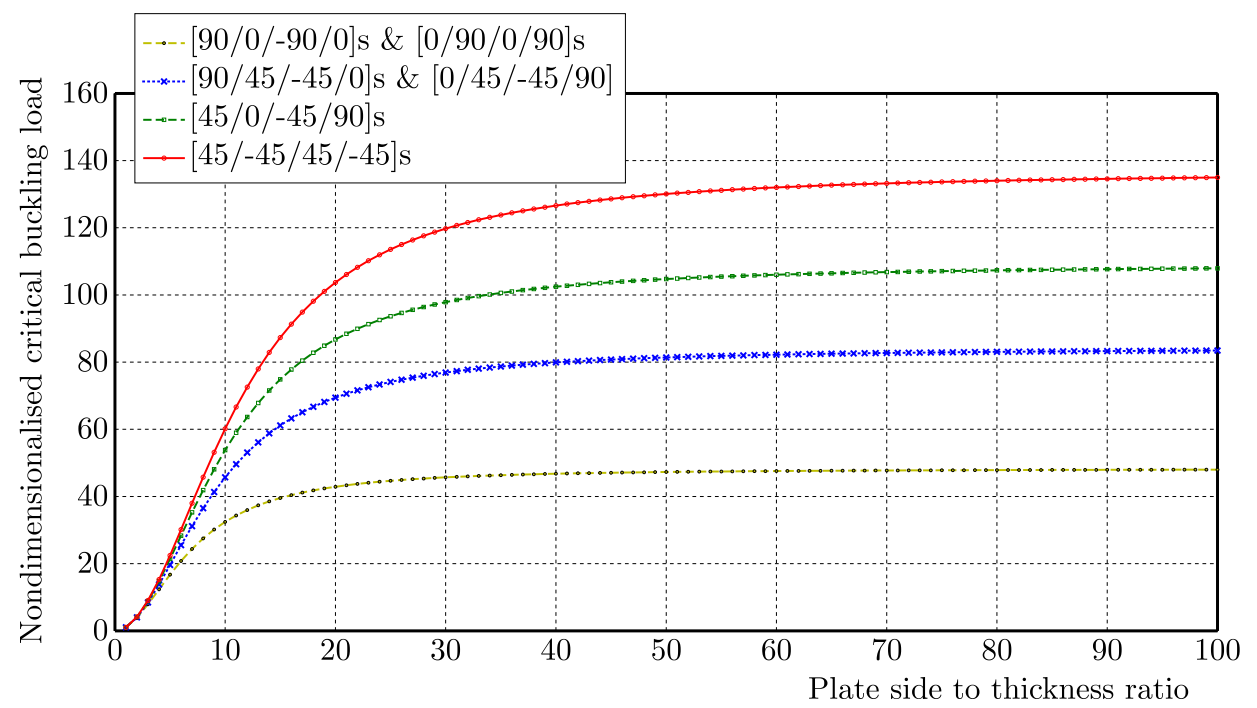

Fig. 3. Curves of the nondimensionalised critical buckling load plotted against the plate side-to-thickness ratio for various stacking sequences for a simply supported plate subjected to uniaxial compression

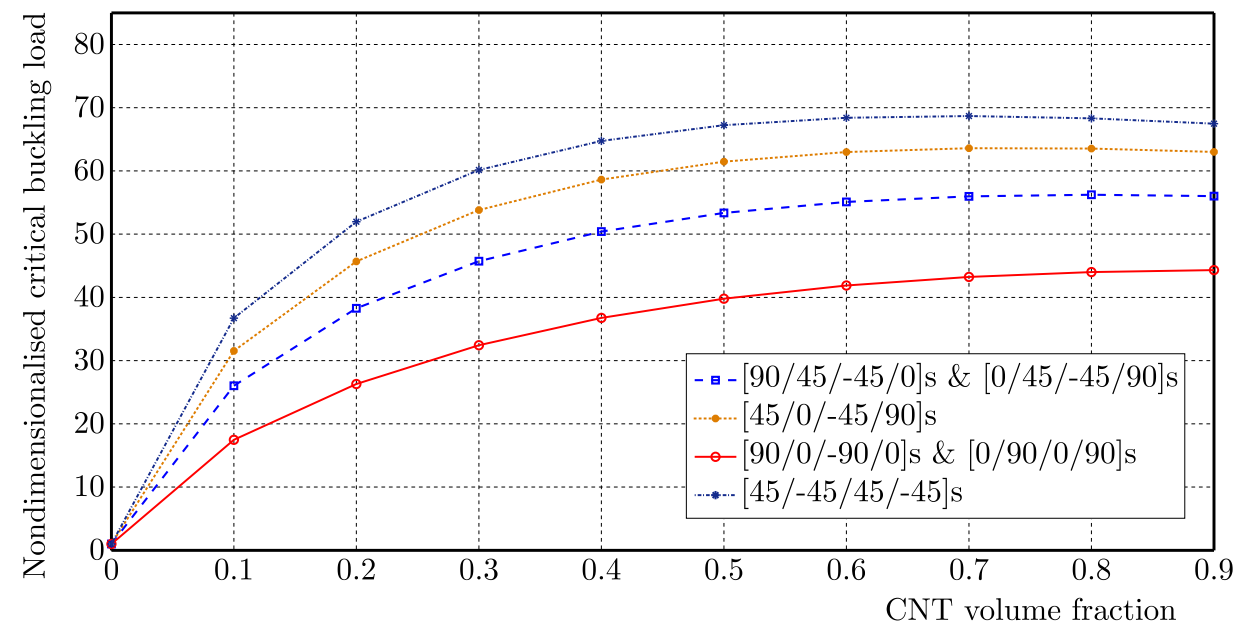

Fig. 4. Curves of the nondimensionalised critical buckling load plotted against the CNT volume fraction for various stacking sequences for a simply supported plate subjected to uniaxial compression

From the above results, it is observed that the angle-ply stacking sequence $[45 /-45 / 45 /-45] \mathrm{s}$ exhibits more resistance to buckling than the cross-ply laminating schemes [0/90/0/90] $\mathrm{s}$ and $[90 / 0 /-90 / 0] \mathrm{s}$ for the same percentage of CNT reinforcement. For all the stacking sequences, the effect of shear deformation on the critical buckling load is clearly observed in thick plates $(0<a / h \leqslant 20)$, and it is insignificant in the case of thin plates $(a / h>20)$ (Reddy, 2004). However, the effect of shear deformation across the thickness on the critical buckling load is the same even the plate is subjected to biaxial compression $(k=1)$ with actual values lesser than those of uniaxial compression $(k=0)$. The comparison between the nondimensionalised critical 
buckling loads of uniaxial and biaxial compression for the stacking sequence $[90 / 45 /-45 / 0] \mathrm{s}$ at CNT volume fraction 0.3 is shown in Fig. 5.

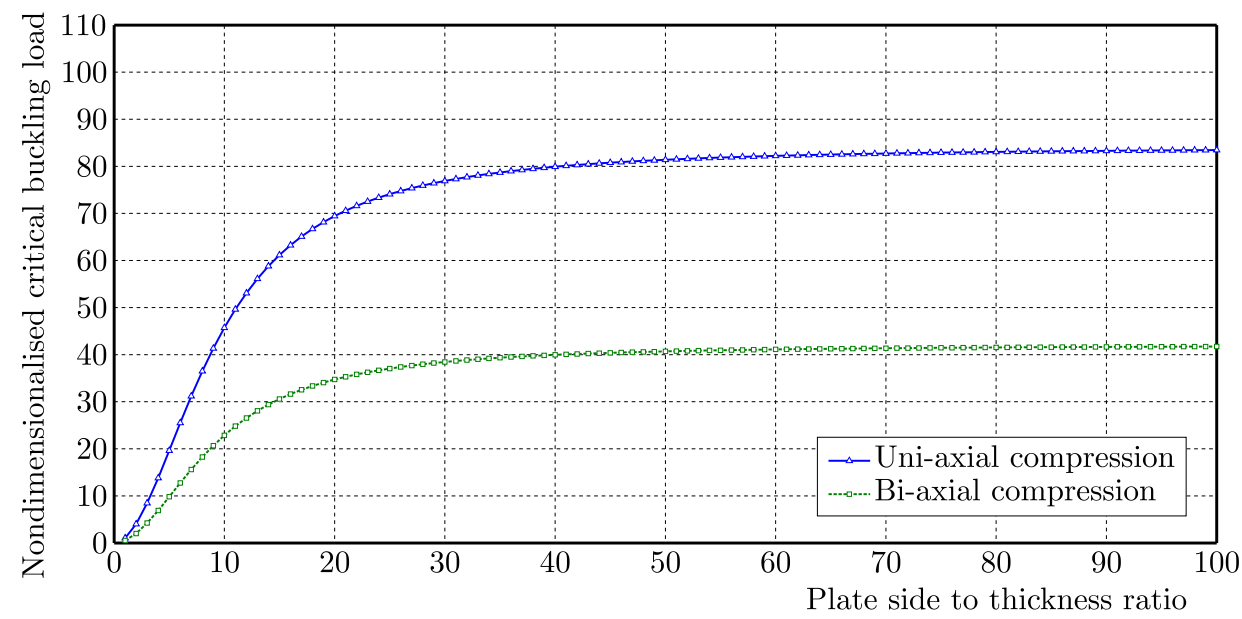

Fig. 5. Curves of the nondimensionalised critical buckling load plotted against the plate side-to-thickness ratio for various loads and stacking sequences $[90 / 45 /-45 / 0] \mathrm{s}$ for a simply supported plate

It is knowledgeable that the nondimensionalised critical buckling load in biaxial compression is about 0.5 times lesser than that of uniaxial compression for various stacking sequences, CNT radii and for various fiber volume fractions. So the results are presented only for uniaxial compression hereafter.

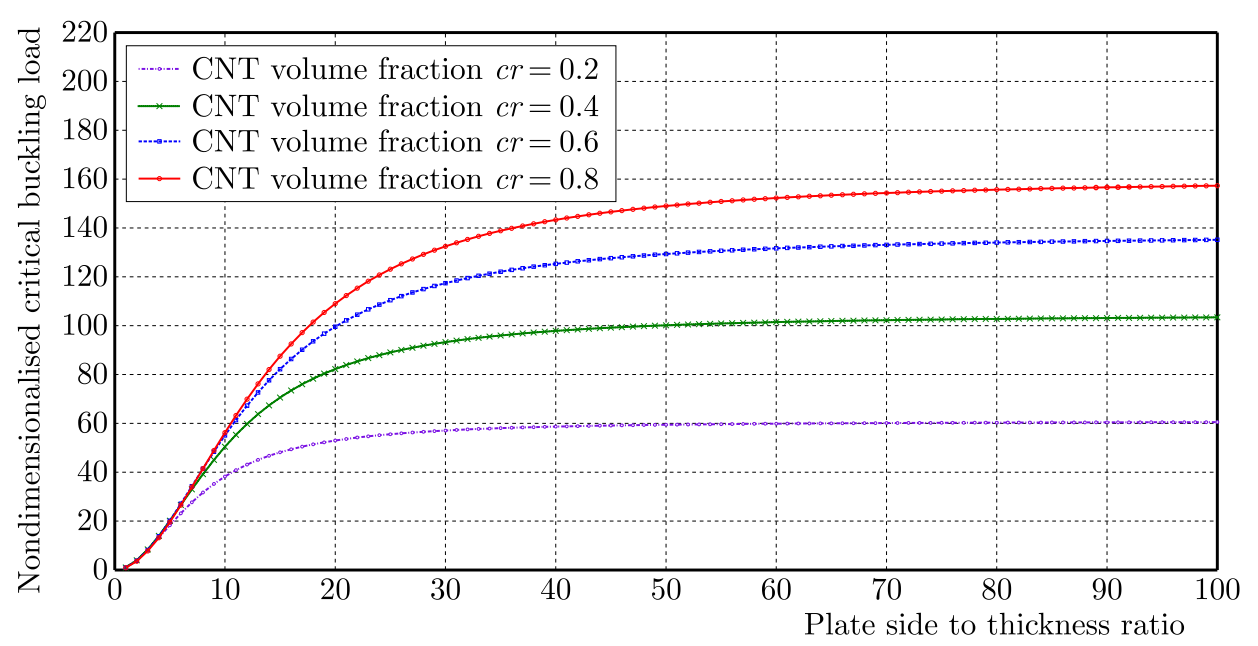

Fig. 6. Curves of the nondimensionalised critical buckling load plotted against the plate side-to-thickness ratio for fiber volume fractions and stacking sequences $[90 / 45 /-45 / 0]$ s for a simply supported plate subjected uniaxial compression

The effect of fiber volume fraction on the critical buckling load is presented in Fig. 6. It is obvious that the critical buckling load increases as the fiber volume fraction in the composite increases, as CNTs are stiffer and stringer. It is further investigated to discover the effect of CNT radius on the critical buckling load, and it is observed from the following graph (Fig. 7) that for the same stacking sequence, the effect of nanotube radius on the critical buckling load issignificant. The same is observed for various stacking sequences, and the results are shown in Fig. 8.

From the above results, it is noticeable that a higher stiffness can be obtained by reinforcing CNTs with smaller diameters and, thereby, a decrease in the critical buckling load can also be achieved. 


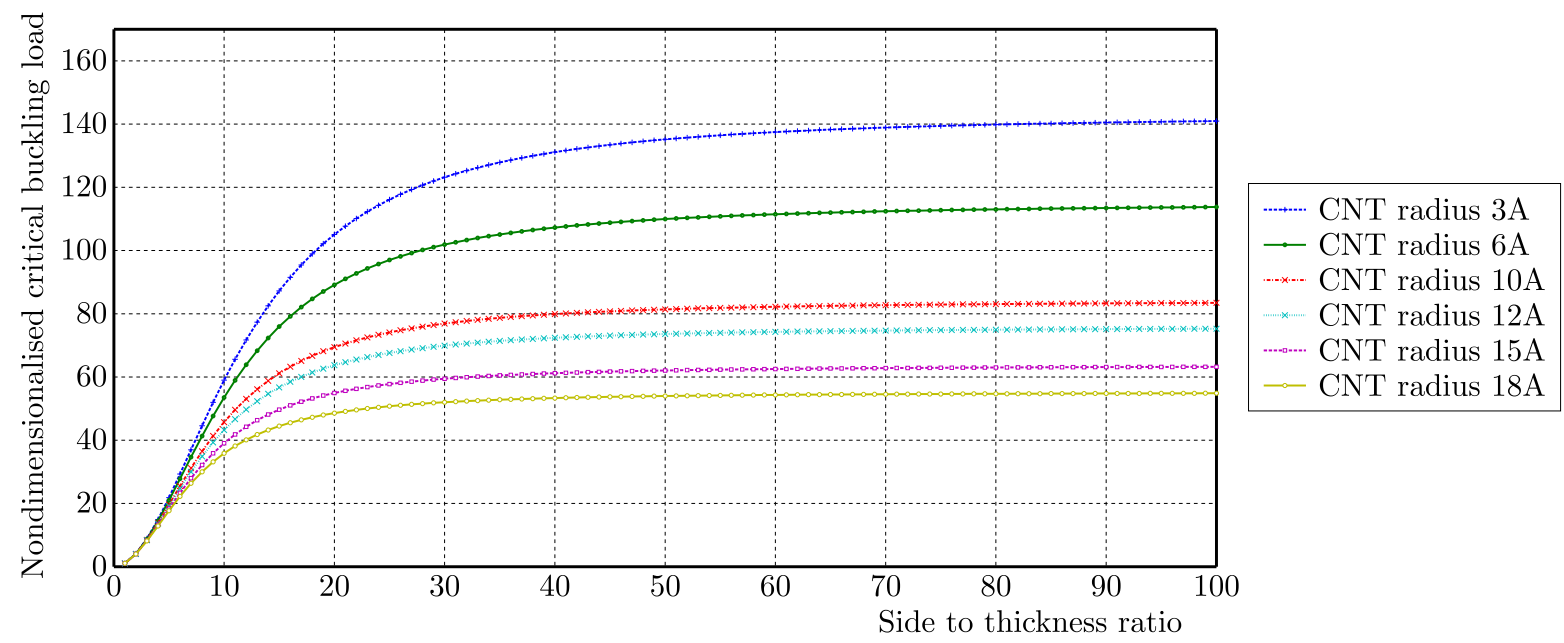

Fig. 7. Curves of the nondimensionalised critical buckling load plotted against the plate side-to-thickness ratio for various CNT radii and stacking sequences $[90 / 45 /-45 / 0] \mathrm{s}$ for a simply supported plate subjected uniaxial compression

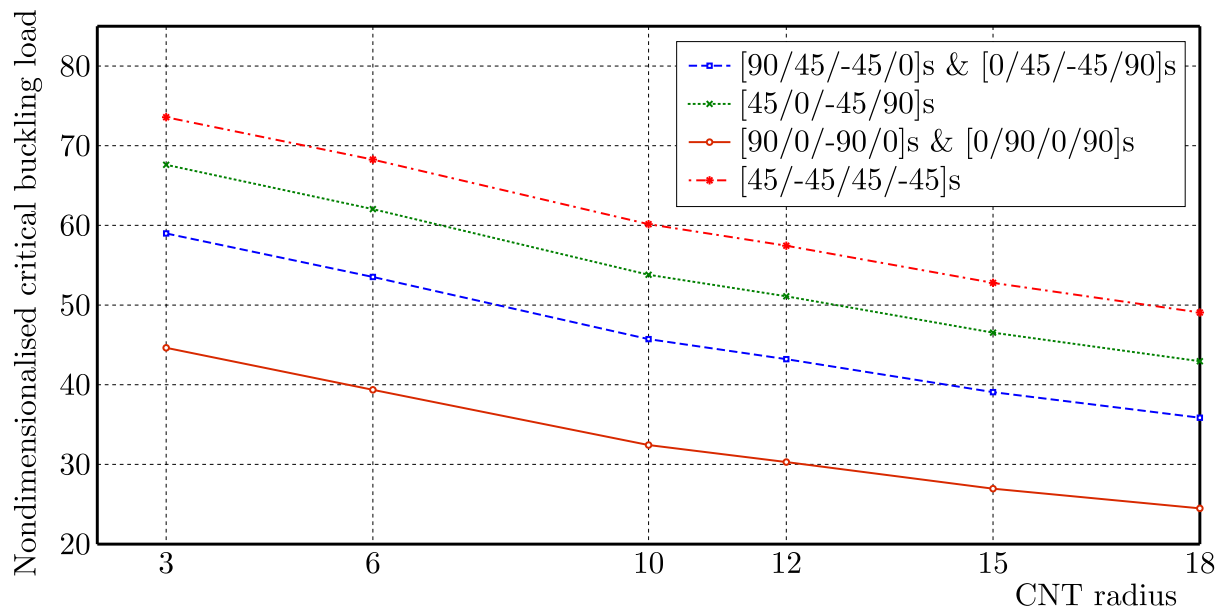

Fig. 8. Curves of the nondimensionalised critical buckling load plotted against CNT radii for various stacking sequences for a simply supported plate subjected uniaxial compression $(k=0)$

\section{Conclusions}

The buckling behavior of a CNT reinforced polymer composite plate is studied by using a MATLAB code. The Mori-Tanaka micromechanics model is adopted for determining the elastic constants of the CNT reinforced polymer composite material in terms of nanotube volume fractions. It is shown that they are in good agreement with the published results. The first order shear deformation theory (FSDT) is considered for determination of the critical buckling loads of plates.

A twofold validation is done to the code; one is to validate the elastic constants of the CNRP composite and the second to validate the FSDT. In the case of CNRP composite plates under FSDT, it is found that the effect of side to thickness ratio on the critical buckling load of thick plates is phenomenal. The results markedly show the diminishing effect of transverse shear deformation on the critical buckling load of thick plates, the effect being negligible for side to thickness ratios greater than 20 i.e., for thin plates. It is also investigated that the stacking sequence of the laminated plate is yet another important parameter for determination of the buckling behavior of the plates. It is observed that the buckling resistance of the angle-ply 
laminating scheme is less than that of other stacking sequences considered in the study. The influence of CNT radii is also examined on the buckling of the plates, and it is proposed to use a smaller radius to obtain a higher buckling resistance. The effect of the volume fraction of the CNT reinforcements on the buckling of the plate is studied, and it is found that the increase in the fiber volume fraction decreases the critical buckling load.

\section{References}

1. Arash B., Wang Q., 2013, Detection of gas atoms with carbon nanotubes, Scientific Reports, 3

2. Arash B., Wang Q., Varadan V., 2014, Mechanical properties of carbon nanotube/polymer composites, Scientific Reports, 4

3. Demczyk B.G., Wang Y.M., Cumings J., Hetman M., Han W., Zettl A., Ritchie R.O., 2002, Direct mechanical measurement of the tensile strength and elastic modulus of multiwalled carbon nanotubes, Material Science Engineering, A334, 173-178

4. Gates T., Odegard G., Frankland S., Clancy T. 2005, Computational materials: multiscale modeling and simulation of nanostructured materials, Composite Science Technology, 65, 15, 2416-2434

5. IIJIma S., 1991, Helical microtubules of graphitic carbon, Nature, 354, 56-58

6. Lau K.-T., Hui D., 2002, The revolutionary creation of new advanced materials - carbon nanotube composites, Composites: Part B, 33, 263-277

7. Madhu S., Subba Rao V.V., Pramod Kumar P., Chandramouli K., 2012, Static and dynamic analysis of carbon nanotube reinforced polymer composite plates, Material science Research Journal, 6, 3-4

8. Ounaies Z., Park C., Wise K.E., Siochi E.J., Harrison J.S., 2003, Electrical properties of single wall carbon nanotube reinforced polyimide composites, Composite Science Technology, 63 $1637-1646$

9. Popov V.N., Doren V.E., Balkanski M., 2000, Elastic properties of crystals of single-walled carbon nanotubes, Solid State Communications, 114, 359-399

10. Reddy J.N., 2004, Mechanics of Laminated Composite Plates and Shells: Theory and Analysis, 2nd edition, CRC Press

11. Saito R., Dresselhaus G., Dresselhaus M.S., 1999, Physical Properties of Carbon Nanotubes, Imperial College Press, London

12. Shi D.L., Feng X.Q., Huang Y.Y., Hwang K.C., GaO H., 2004, The effect of nanotube waviness and agglomeration on the elastic property of carbon nanotube-reinforced composites, Journal of Engineering Materials and Technology, 126, 250-257

13. Silani M., Talebi H., Ziaei-Rad S., Kerfriden P., Bordas S.P., Rabczuk T., 2014, Stochastic modelling of clay/epoxy nanocomposites, Composite Structures, 118, 241-249

14. Tserpes K.I., Papnikos P., 2005, Finite element modelling of single-walled carbon nanotubes, Composites: Part B, 36, 468-477

15. Wang Q., 2008, Atomic transportation via carbon nanotubes, Nano Letters, 9, 1, 245-249

16. Weisenberger M.C., Grulke E.A., Jacques D., Rantell T., Andrews R., 2003, Enhanced mechanical properties of polyacrylonitrile/multiwall carbon nanotube composite fibers, Jorunal of Nanoscience Nanotechnology, 3, 6

17. Wuite J., Adali S., 2005, Deflection and stress behaviour of nanocomposite reinforced beams using a multiscale analysis, Composite Structures, 71, 3-4, 388-396 
18. Xiao J.R., Gama B.A., Gillespie JR J.W., 2005, An analytical molecular structural mechanics model for the mechanical properties of carbon nanotubes, International Journal of Solids Structures, 42, 3075-3092

19. Zhang Y., Zhao J., Wei N., Jiang J., Gong Y., Rabczuk T., 2013, Effects of the dispersion of polymer wrapped two neighbouring single walled carbon nanotubes (swnts) on nanoengineering load transfer, Composites: Part B Engineering, 45, 1, 1714-1721

20. Zhang Z., Liu B., Huang Y., Hwang K., GaO H., 2010, Mechanical properties of unidirectional nanocomposites with non-uniformly or randomly staggered platelet distribution, Journal of Mechanics and Physics of Solids, 58, 10, 1646-1660

Manuscript received July 25, 2016; accepted for print July 18, 2017 